\section{Migräneexperten leiden an Migräne}

Wissenschaftler der Universität Houston stellten fest, dass Neurologen öfter an $86 \%$ der weiblichen und $61 \%$ der männlichen befragten Spezialisten haben oder hatten schon eine episodische Migräne. Für die US-Bevölkerung haben Umfragen eine Lebenszeitprävalenz von lediglich $43 \%$ bei Frauen und $18 \%$ bei Männern ergeben.

Evans RW et al. Headache 2016

\section{Synkope erhöht Unfallrisiko}

Dänische Wissenschaftler haben für eine Kohortenstudie die Daten von mehr als vier Millionen erwachsenen Dänen analysiert. Rund 41.000 Personen hatten zwischen 2008 und 2012 eine Synkope erlitten. In den folgenden zwei Jahren waren 4,4\% dieser Patienten an einem Verkehrsunfall beteiligt und mussten deswegen ärztlich versorgt werden. Die Inzidenzrate lag mit 20,6 pro 1.000 Personenjahre (PJ) fast doppelt so hoch wie in der Allgemeinbevölkerung mit 12,1/1.000 PJ.

Numé AK et al. JAMA Intern Med 2016

\section{Helfen Kohlwickel bei Arthrose?}

In einer Studie wurde die Wirkung des Kohlwickels bei Kniegelenksarthrose untersucht. Verglichen wurde der Krautwickel mit der Anwendung von DiclofenacGel bzw. einer Fortführung der bisherigen Therapie (UC-Gruppe). Hatten zu Beginn der Studie alle die Schmerzintensität ähnlich eingeschätzt, gab die Gruppe, die Krautwickel aufgelegt hatte, nach vier Wochen weniger Schmerzen an als die UCGruppe. Zwischen Krautwickeln und Diclofenac gab es keinen Unterschied.

Lauche Ret al. Clin J Pain 2016. Kopfschmerzen und Migräne leiden

Neue Perspektive

\section{Rauchstopp mit dem Freiburger Modell}

Ein neues Konzept der stationär-ambulanten Kooperation wird mit dem Freiburger Modell effizient umgesetzt. Eine Evaluation der ersten zwei Jahre zeigt sehr gute Ergebnisse.

Trotz der großen Zahl an Rauchern in Deutschland und des flächendeckenden Angebotes von Therapiemöglichkeiten, werden diese nur sehr selten wahrgenommen. Aus der Erfahrung landen $\mathrm{Pa}$ tienten trotz starken Interesses leider nur selten in einem Entwöhnungsprogramm. Das Präventionsteam des Tumorzentrums der Uniklinik Freiburg wurde 2008 mit der Aufgabe etabliert, rauchenden Patienten die Entwöhnung zu erleichtern. Hierzu werden die Patienten an unabhängige, externe Anbieter von Thera- pieprogrammen vermittelt und von Beginn bis Ende der Therapie intensiv begleitet, um die Compliance und die Nachhaltigkeit sicher zu stellen. In einer Evaluation der ersten zwei Jahre nach Einführung des Angebotes wurden Daten von 1.432 Teilnehmern ausgewertet. Eine durchgehende Abstinenz konnte bei $28 \%$ erreicht werden. 7-Tage Punkt-Prävalenzen für 3, 6 und 12 Monate nach der Beratung lagen zwischen 30 und 35 Prozent. Die Abstinenzraten erscheinen umso bemerkenswerter, wenn man bedenkt dass, ausschließlich Patienten teilnahmen, die nicht primär mit dem Wunsch einer Raucherentwöhnung an die Klinik herangetreten waren. (J.Leifert)

SpringerMedizin.de, 2.3.2016

\section{Zusammenhang mit Asthma}

\section{Paracetamol in der Schwangerschaft}

Kinder, die im Mutterleib oder als Säuglinge Kontakt zu Paracetamol hatten, leiden öfter an Asthma. Ursache scheint der Wirkstoff und nicht die behandelte Erkrankung zu sein.

Ärzte aus Norwegen und Großbritannien haben den Zusammenhang zwischen Asthmainzidenz und der Behandlung mit Paracetamol in utero und im Säuglingsalter genauer beleuchtet. Sie sammelten Daten von aktuell an Asthma erkrankten Kindern im Alter von drei (von 53.169 Kindern) und sieben Jahren (von 25.394 Kindern). Bei ausschließlich pränatalem Paracetamolkontakt lag die Asthmarate bei Dreijährigen um 13\%, bei Siebenjährigen um 27\% höher als bei nicht exponierten Kindern. Ein Kontakt im ersten Lebenshalbjahr ging mit 29\% bzw. 24\% höherem Risiko einher. Die Exposition in beiden Phasen war mit Steigerungen von $27 \%$ bzw. $49 \%$ verbunden. Bei der Risikoberechnung wurden Asthma, Atemwegsinfekte, Fieber, Schmerzen und Antibiotikagebrauch in Schwangerschaft und Stillzeit berücksichtigt. Die Assoziation erwies sich als unabhängig davon, zu welcher Indikation der Wirkstoff angewendet wurde. Wegen der großen Bedeutung von Paracetamol als Analgetikum/ Antipyretikum für Schwangere und Säuglinge „sei die Entdeckung potenzieller Nebenwirkungen von erheblichem Belang für die öffentliche Gesundheit“, so Magnus und Kollegen. Eine Überprüfung in randomisierten Studien sei daher wünschenswert. 